\title{
Revertiamo a rivertire o adottiamo revertare?
}

\author{
Anna M. Thornton
}

PUBBLICATO: 7 SETTEMBRE 2021

\section{Quesito:}

Diversi lettori chiedono informazioni su quale sia la forma italiana corrispondete al verbo inglese to revert, che ha usi tecnici in biologia e in informatica. I lettori citano revertire per l'uso in biologia, e revertare per l'uso in informatica, ma dichiarano di non aver trovato questi verbi nei dizionari.

\section{Revertiamo a rivertire o adottiamo revertare?}

$\mathrm{E}$ ffettivamente, le forme proposte dai lettori non si trovano in alcuni dei più comuni dizionari di riferimento dell'italiano, come lo Zingarelli 2020 e il DISC, ma allargando la ricerca al Nuovo De Mauro e al GDLI alcune prime risposte si trovano.

Il Nuovo De Mauro lemmatizza rivertire con un rimando secco a rivertere, che dichiara attestato dall'inizio del XIV secolo, con due usi qualificati come oggi obsoleti, 'trasformare, mutare' e 'piegare, rivolgere allinsù', e un uso tecnico-scientifico della biologia 'ritornare allo stato originario dopo una mutazione genetica', che è proprio l'uso sul quale verteva la domanda del lettore Dario A.

Il GDLI lemmatizza il tipo come "rivertere e rivertire", e articola più dettagliatamente del Nuovo De Mauro le diverse accezioni. L'accezione più antica è 'trasformare, mutare una cosa in un'altra' (per es. de dona in serpe fo reversa 'fu trasformata da donna in serpente', Poesie musicali del Trecento, LXXXIV38), seguita poi da 'piegare, rivolgere all'insù o all'ingiù una parte del corpo', senso esemplificato con i versi danteschi "La grave idropesi, [...] / faceva lui tener le labbra aperte / come l'etico fa, che per la sete / l'un verso 'l mento e l'altro in sù riverte" (Inf. XXX, 52-57). Il GDLI registra poi un senso generico già antico, 'tornare a uno stato, in una condizione precedente', che documenta con una citazione dalla Bibbia volgare, testo toscano del XIV-XV secolo: "Tutte le cose che sono di terra, in terra rivertiranno". Infine, come ultima accezione in senso diacronico, il GDLI segnala l'uso tecnico in biologia 'ritornare allo stato originario dopo una mutazione genetica', documentato con un'attestazione giornalistica tratta dalla "Stampa" del i986:

si tratta cioè del vaccino attenuato ideale, che non reverte mai, quello che si è sempre cercato di ottenere mediante le tecniche di attenuazione tradizionali.

Una ricerca in rete permette di confermare un uso tecnico proprio della biologia, 'ritornare allo stato originario', non necessariamente limitato a reversioni dopo mutazioni genetiche. Ad esempio, nel Bollettino della Societá italiana di biologia sperimentale, I958, p. I327, si legge:

L'azione dell'istamina reverte con alcuni lavaggi, ed è soppressa dal pretrattamento con esametonio 4.10 $\mathrm{IO}^{-4}$.

Un'attestazione recente si ha nel manuale Microbiologia medica ( $8^{\text {a }}$ ed.) di P. R. Murray, K. S. Rosenthal e M. A. Pfaller (Milano, Edra, 20I7), tradotto dall'inglese, nel quale si legge: 
I maggiori svantaggi del vaccino ottenuto da virus vivi sono i seguenti: (I) il virus vaccinico può infettare un individuo immunologicamente compromesso; (2) esiste una remota possibilità che il virus reverta alla sua forma virulenta e causi la forma di malattia paralitica.

Si noti che i dizionari lemmatizzano rivertere o rivertire, mentre sia i lettori che hanno posto quesiti sia gli esempi appena citati attestano forme con $r e^{-}$, non con $r^{-}$. L'alternanza tra re- e ri- è comunque attestata fin dall'inizio, come si vede dagli esempi antichi citati dal GDLI.

Dalle forme che ricorrono negli esempi del senso tecnico in biologia non è possibile dedurre con certezza se l'infinito corrispondente sia revertere o revertire: i diversi verbi italiani che risalgono a una base latina vertěre sono soprattutto in -ire, ma ve ne sono anche in -ere. I verbi in -vertire elencati nel Nuovo De Mauro sono avvertire, convertire, divertire, invertire, pervertire, sovvertire e loro prefissati (riconvertire, ecc.), e hanno tutti coniugazione senza inserzione dell'elemento -isc- presente nella maggioranza dei verbi in -ire: le forme reverte, reverta viste negli esempi citati sono quindi compatibili sia con un infinito revertire che con un infinito revertere, dato che i verbi in -ere e quelli in -ire senza -isc- hanno le stesse desinenze nella terza singolare del presente indicativo e congiuntivo (teme, legge, tema, legga come sente, dorme, senta, dorma); in -vertere il Nuovo De Mauro elenca una serie di lemmi per lo più obsoleti o di basso uso (avertere, controvertere, estrovertere, evertere, introvertere, prevertere), oltre al nostro rivertere e a vertere. In questo quadro, si comprende il dubbio dei lettori: nel loro uso il verbo in questione consapevolmente corrisponde all'inglese revert, e questo spiega la preferenza per la forma con re- invece che ri-; la consapevolezza che -vert risale anch'esso al latino vertère, e che verbi con questo etimo in italiano sono per lo più in -ire (convertire, ecc.), produce revertire, la forma proposta dai lettori. I dizionari ammettono forme sia in -ere (preferito) sia in -ire, ma con ri- invece che re-, il quale è invece favorito o almeno diffuso nell'uso, oggi per maggiore aderenza alla forma inglese revert e in antico per maggiore aderenza alla forma latina revertere.

Il verbo presenta quindi molteplici cause di incertezza: re- o ri-, -vertere o -vertire?

La questione si complica ulteriormente se consideriamo anche la forma revertare, non lemmatizzata nei dizionari consultati, ma segnalata, insieme a molti altri adattamenti di verbi inglesi usati nel lessico tecnico dell'informatica e del mondo dei giochi di ruolo, dal lettore Mauro P., che elenca killare, pushare, committare, revertare, fixare, discoverare, hashare, bildare (presumibilmente da build 'costruire', con adattamento ortografico), jumpare, switchare. Come si vede, in questi casi l'adattamento dei prestiti produce verbi della prima coniugazione, come avviene normalmente, anche in verbi di uso non solo tecnico, come chattare, cliccare, stoppare (per esempi nel lessico dei giochi di ruolo si veda qui).

Sarebbe buona norma cercare di rendere questi termini tecnici con una parola italiana corrispondente, ma l'adozione dei prestiti adattati, inseriti nella prima coniugazione, è la pratica più diffusa. In questo caso, però, la disponibilità di revertere o rivertere potrebbe fare la differenza. D'altra parte, potrebbe anche affermarsi una differenziazione funzionale tra revertere o revertire (come abbiamo visto, le forme con re- invece che ri- sembrano decisamente prevalenti) nell'ambito della biologia, dove la voce è usata in continuità con il senso documentato già in italiano antico 'tornare a uno stato originario', e revertare nell'ambito dell'informatica, dove la voce (peraltro di scarsissima diffusione) è percepita come adattamento di un prestito dall'inglese, senza che se ne colga la relazione né con il latino revertere né con forme e sensi attestati in italiano antico. 


\section{Cita come:}

Anna M. Thornton, Revertiamo a rivertire 0 adottiamo revertare?, "Italiano digitale", XVIII, 2021/3 (luglio-settembre)

DOI: $10.35948 / 2532-9006 / 2021.10599$

Copyright 2021 Accademia della Crusca

Pubblicato con licenza creative commons CC BY-NC-ND 